\title{
The QBIT theory of consciousness
}

\author{
Majid Beshkar \\ Tehran University of Medical Sciences \\ mbeshkar@sina.tums.ac.ir \\ majid.beshkar@gmail.com
}

\begin{abstract}
This paper introduces the first version of the QBIT theory of consciousness which is based on inspirations and insights from Quantum mechanics, Biology, Information theory, and Thermodynamics. The ultimate goal of the QBIT theory is to find scientifically correct answers to the following four questions: What is the nature of qualia? How are qualia generated? Why are qualia subjective? Why does a quale have a particular meaning?

The theory, in its first version, proposes tentative answers to these four fundamental questions about consciousness. According to the QBIT theory qualia are quantum information encoded in maximally entangled pure states. Qualia are generated when robustness of quantum information exceeds a certain threshold. Qualia are subjective because maximally entangled pure states are not shareable. The quality or meaning of a particular quale is determined by its associated internal model.
\end{abstract}

Keywords: Consciousness; Entanglement; Information; QBIT; Qualia; Quantum; Thermodynamics 


\section{Introduction}

The problem of consciousness is one of the most difficult problems in biology, which has remained unresolved despite several decades of scientific research. The hard core of the problem of consciousness is in fact the problem of qualia.

Qualia (plural for quale) is a technical term that refers to subjective conscious experiences or raw feels such as a red color, a particular smell, a specific taste, and so on. As an example, when we see a red flower, the redness that we feel is a quale. For this reason, some authors prefer to use the term "atomic microfeels" instead of qualia. ${ }^{1}$ Qualia are the building blocks of consciousness. Our consciousness at any moment consists of several qualia. "To be conscious" means "to have qualia", and unconscious experience means "qualialess experience".

The QBIT theory is a new approach to the problem of consciousness based on inspirations and insights provided by Quantum mechanics, Biology, Information theory, and Thermodynamics. The theory formulates the problem of consciousness in the following four questions: What is the nature of qualia? How are qualia generated? Why are qualia subjective? Why does a quale have a particular meaning?

This paper introduces the first version of the QBIT theory, and proposes tentative answers for each of the four fundamental questions mentioned above. In sum, it is conjectured that a quale is a dense pack of meaningful quantum information encoded in maximally entangled pure 
states. Subjectivity of qualia are supposed to be a consequence of the fact that maximally entangled pure states are unshareable. Furthermore, it is hypothesized that the specific quality or meaning of a quale is determined by the activity of an associated internal model embedded in the system.

\section{Consciousness requires quantum entanglement}

The QBIT theory proposes that when certainty of an observer about an event exceeds a certain level, the observer becomes conscious of that event. To achieve this high level of certainty, quantum information is required. As Patrick Hayden $^{2}$ nicely mentions, "with quantum information, it is possible not just to be certain, but to be more than certain." This wonderful effect of quantum mechanics inspires the idea that in order to become conscious, we need to go beyond the classical limits. Consciousness requires quantum information.

Information, entropy (or uncertainty), and correlation are three distinct, but intimately related, concepts in classical (or Shannon) information theory. When an agent observes (or measures) a system, a correlation is established between the agent and the system. As a result of this correlation, the agent obtains information about the system. As the strength of correlation is increased, the amount of information also increases, and entropy (or uncertainty of the agent about the system) decreases. ${ }^{3-5}$ 
To achieve very high levels of certainty, a very strong kind of correlation (or a supercorrelation) is required. Such supercorrelations are only possible in quantum (and not classical) physics. In fact, quantum entanglement is a kind of supercorrelation ${ }^{6}$ which, according to the QBIT theory, is necessary for consciousness.

Decoherence is a common obstacle for all phenomena that depends on the capacity of preserving and using quantum entanglement and coherence. ${ }^{7}$ Typically, decoherence rapidly destroys coherence in a quantum system and the entanglement between its subsystems. ${ }^{8}$ However, there is strong evidence that entanglement and coherence play significant roles in certain biological processes, including photosynthesis $^{9}$ and bird navigation. ${ }^{10}$ These unique quantum phenomena could also occur in the human brain to support consciousness.

\section{Consciousness requires quantum computation}

Although centuries of philosophical dualism have led us to consider consciousness as unreducible to physical interactions, scientific evidence is compatible with the proposition that consciousness arises from nothing more than a particular type of computation. ${ }^{11}$ According to the QBIT theory, this particular type of computation is quantum computation.

Some unique and wonderful effects of quantum computation arise only when maximally entangled pure states are available 
for use. In fact, maximally entangled pure states are ideal resources for quantum computation, while mixed states are not very useful for this purpose. ${ }^{12}$

From a thermodynamic point of view, production of pure maximally entangled states is costly, meaning that it requires consumption of energy and production of entropy. However, for some computational tasks such as estimating a given parameter with a high precision, it is more cost effective for a system to use maximally entangled pure states rather than using already available mixed states. ${ }^{13}$ Therefore, above a certain level of precision, the cost of computation will be reduced if maximally entangled pure states are used. These arguments inspire the idea that consciousness might also be dependent on computations that require maximally entangled pure states.

But in the warm and noisy environment of a biological system, only mixed states are readily available due to imperfection of operations and the effects of decoherence. What are the mechanisms by which a biological system (such as the human brain) deals with the noise and decoherence so that it can take advantage of the interesting features of maximally entangled pure states? Stuart Hameroff ${ }^{14}$ suggests that there are at least two anti-decoherence mechanisms in the brain: (1) encasing bundles of microtubules in actin gel, which provides a shielded and isolated environment for quantum computation; (2) microtubule quantum error correction topology. The QBIT theory suggests dynamical 
decoupling as another anti-decoherence mechanism in the brain and other biological systems. Dynamical decoupling could extend the lifetime of an entangled state. ${ }^{15}$ Evidence shows that dynamical decoupling can be achieved using recurrent neural networks. ${ }^{16,17}$

Since consciousness requires maximally entangled pure states, a conscious agent should be endowed with a mechanism that constantly produces such states. For this reason, the QBIT theory proposes that consciousness requires Maxwell demon-assisted quantum computation. In thermodynamics, a Maxwell demon is a mechanism or a machine that extracts work and removes heat from a target system. Work extraction and heat removal (which is a thermodynamic process) is equivalent to converting mixed states to pure states (which is an information theoretic process). ${ }^{18}$

In quantum mechanics, there is a special kind of Maxwell demon which is able to transition a qubit into a purer state. ${ }^{19}$ In other words, it can inject pure states to an ongoing quantum computation. This kind of Maxwell demon is in sharp contrast to locally operating classical Maxwell demons. It can purify a target qubit over macroscopic distances on the order of meters and tolerates elevated temperatures of the order of a few Kelvin. Such a spatial separation between the system and the demon has practical benefits because it prevents undesired heating of the system during the demon's memory erasure. In fact, this particular demon not only 
purify a qubit but also makes the environment surrounding the qubit slightly colder. Furthermore, in contrast to the classical demon, this quantum demon utilizes its purity or coherence as a thermodynamic resource. The quantum demon does not measure the state of the target system; therefore, its operation is rooted in purity rather than information. ${ }^{19}$

Although the concept of Maxwell demon was first introduced in thermodynamics, it gradually found applications in other scientific disciplines including information theory and biology. In biology, for example, it has been demonstrated that the action of a Maxwell demon is necessary for chemotaxis in Escherichia coli. ${ }^{20,21}$ In the case of chemotaxis in Escherichia coli, the Maxwell demon attempts to reduce the effects of the environmental noise on the target system. The QBIT theory proposes that consciousness is another biological process in which Maxwell demons play important roles. One of these roles is production and preservation of maximally entangled pure states for quantum computation. The other possible role is compression of information to make a dense pack of information.

\section{Why does a quale have a particular meaning?}

Information on its own has no intrinsic meaning. It is "interpretation" that adds meaning (or semantic content) to information. The same bit of information can have different meanings, depending on how it is interpreted. ${ }^{22}$ The QBIT 
theory proposes that what interprets information is an internal model which is encoded in the system. For each quale that a system can generate, there is an associated internal model encoded in the system. The quality or meaning of a quale (for example, painfulness or redness) is thus determined by its associated internal model.

Internal models are representations of the environment within a system. In other words, internal models are symbols that represent external realities. For example, the internal model for a red quale is a symbol that represents all electromagnetic waves with a wavelength of 635-700 nm. Therefore, internal models could be regarded as mediators between the outside world and internal cognitive processes. ${ }^{23}$ They transform information into usable formats for the system.

To generate a quale, its associated internal model should be activated first. Activation or stimulation of an internal model reduces uncertainty and generates semantic information. If robustness of this information exceeds a certain threshold, it is experienced as a quale. If not, activation of the internal model results in a quale-less (or unconscious) experience. Even in the absence of consciousness and sensory inputs, internal models can guide the behavior of a system. ${ }^{24}$ Internal models may or may not be necessary for intelligent behavior; however, they are absolutely necessary for consciousness. A system without an internal model cannot be conscious, no matter how intelligent its behavior is. 
An internal model is proposed to be a specialized pattern of network activity that is formed as a consequence of adaptive processes. Internal models are created as a cognitive system observes and interacts with its environment for a long enough time. In other words, internal models gradually form as the system repeatedly acts on the environment through its actuators and receives feedback through its sensors. ${ }^{24}$ When the environment or the tasks that should be performed to survive in the environment are complex enough, the cognitive system reacts to this challenge by developing internal models. ${ }^{24}$ Expectations and needs are two factors that shape internal models of an animal. As animals evolve to behave appropriately and survive in a dynamic environment, internal models of the environment emerge within their nervous systems. Internal models are flexible. They could be shaped by learning, and become updated during the lifetime of an animal or over the course of evolution. ${ }^{24}$

Where are internal models encoded in the human brain? In the case of shape perception, internal models are probably encoded in the lateral occipital complex..$^{25,26}$ In the case of color qualia, evidence shows that, while cortical area V1 plays an essential role in color perception, it does not contain representations (or internal models) corresponding to specific colors. It is in the area V2 that the first evidence for hue maps (i.e., red, green, blue, purple, and so on) emerges. Area V4 has color-tuned neurons, but it is unclear whether these neurons inherit their color preferences or compute 
them within $\mathrm{V} 4 .{ }^{27}$ Therefore, in the human brain, the internal model for a red quale might be encoded in the area V4 or distributed across several brain areas. No matter where and how it is encoded, activation of this internal model is necessary (although not sufficient) for a human to experience a red quale.

\section{How are qualia generated?}

The QBIT theory hypothesizes that stimulation of an internal model generates semantic information and reduces uncertainty. The more it is stimulated, the more information is generated. When a lot of information is generated and packed in a small volume, a quale emerges. In a more technical language, a quale is generated when robustness of information exceeds a certain threshold. The robustness of information is determined by both the amount and the density of information. In fact, robustness of quantum information implies how many qubits are packed in a given volume. When semantic quantum information becomes robust enough, it transforms into a quale.

Internal models could be activated by external stimuli through the sensors, or activated internally by attentional mechanisms. In the QBIT theory, attention is regarded as a mechanism that stimulates internal models to generate more information. In fact, attention amplifies information. Information amplification means increasing the amount of information. The global neuronal workspace theory proposes 
that consciousness emerges due to the amplification of information. ${ }^{28}$ However, according to the QBIT theory, generating huge amounts of information does not necessarily give rise to emergence of consciousness, unless information is packed into a small enough volume.

It should be emphasized that not all quantum information encoded in maximally entangled pure states are qualia. To generate a quale, three conditions should be met. First, an internal model should be activated to generate semantic information. Second, this information should be encoded in maximally entangled pure states. Third, information should be amplified and packed densely enough. Experimental evidence shows that visual consciousness emerges between 180 and 230 ms after the onset of a visual stimulus. ${ }^{29}$ Most of this time delay is probably due to interpretation and amplification of information.

\section{Why are qualia subjective?}

A prime feature of qualia is that they are subjective. This means that they are private and unshareable, accessible only to the system that is generating them. Observation or measurement of qualia generated within a system is not possible for any other system.

"Entanglement is the quantum equivalent of what is meant by privacy." ${ }^{12}$ This nice statement, and the argument behind it, in a paper by the Horodecki family provided insight for the QBIT theory that quantum entanglement might be able 
to explain the subjectivity of consciousness. Quantum entanglement has limited shareability. In the case of pure states, it can even be absolutely unshareable. ${ }^{30}$

A quale could be regarded as a private key. In terms of information theory, a private key is a string of bits which has two important features. First, it is perfectly correlated. Second, it is inaccessible to any other person. ${ }^{12}$ The first feature is due to maximal entanglement. The second feature is due to maximal coherence (or purity), because an eavesdropper who attempts to obtain knowledge about the private key will unavoidably disturb it, introducing a phase error into the system, which destroys purity. ${ }^{12}$

All these arguments can be expressed in terms of the monogamy of entanglement. According to the monogamy of entanglement, maximally entangled pure states are not shareable. ${ }^{31,32}$ Since qualia are encoded in maximally entangled pure states, they should be private and unshareable. This explains why the QBIT theory proposes that subjectivity of consciousness is a consequence of entanglement monogamy.

\section{Conclusion}

The QBIT theory proposes that qualia are quantum information in nature, and emergence of qualia requires quantum computation. Most physical phenomena in nature can be formulated and better described in terms of information processing. ${ }^{33}$ Gravity is a prominent example. 
Reconciling quantum mechanics with gravity is a hard and yet unresolved problem in physics. Recently, quantum information theory and concepts like entanglement and quantum error correction have come to play a fundamental role in solving this problem. For example, it has been suggested that gravity comes from quantum information. ${ }^{34}$ Furthermore, recent evidence from theoretical physics imply that entangled qubits are not only the origin of gravity, but also the origin of matter and space. ${ }^{35}$ It seems that, at some level, everything reduces to information. ${ }^{36}$ This inspires the QBIT theory to propose that, at a fundamental level, qualia are quantum information or entangled qubits. As our knowledge about the nature of quantum information increases, we would gain more insights about the nature of qualia. At present, we know that quantum information is nonlocal. It does not make sense to ask where quantum information is at any given time; it is nonlocally distributed in the entangled state. ${ }^{32}$ Since quantum information is nonlocal, qualia should also be nonlocal. Furthermore, there is some evidence that quantum information is physical. ${ }^{37}$ If this turns out to be true, then qualia must also be physical.

The fact that we have qualia strongly suggests that qualia must have some reason to exist. ${ }^{38}$ One probable reason is that each quale packs a lot of information in a concise and usable format. In other words, a quale is a convenient summary of a huge amount of information. ${ }^{38} \mathrm{~A}$ cognitive system can perform sophisticated tasks without generating qualia, but 
qualia might be necessary to perform some tasks that require greater speed and precision.

To generate qualia, the presence of internal models is necessary. A cognitive system that is endowed with internal models always has some prior information (or partial information) about the outside world. Therefore, the system inevitably looks at the environment through the eyeglasses of internal models. From an information theoretic point of view, partial information is equal to conditional entropy. ${ }^{12}$ In other words, an internal model could be regarded as the shared (or mutual) entropy between the system and its environment, given the states of the sensors (i.e., conditioned on the sensors). Therefore, an internal model is that part of the shared entropy between the system and the environment that goes beyond what is seen in the sensors. ${ }^{24}$ In other words, internal models are information that a system already has about its environment above and beyond what is currently provided by its sensors. ${ }^{39}$ This concept opens up a door for further exploration and extension of the QBIT theory. This extension requires a detailed discussion of the possible relationship between the concept of internal model and the concepts of mutual information and conditional entropy. This is the aim of a future paper.

According to the QBIT theory, a specialized type of Maxwell demon is necessary for consciousness. Throughout evolution, nature has created a variety of different Maxwell demons, each for a specific purpose. These Maxwell demons are 
probably responsible for the emergence of life, evolution of biological organisms, and emergence of consciousness.

In the evolution of the universe, emergence of life could be thought of as a link in the long chain of matter upgrading events. ${ }^{40}$ Emergence of life means emergence of living matter. The amazing complexity of living matter might be the outcome, over long evolutionary timescales, of a great work extraction process (or Maxwell demon) used to create new high-free-energy structures suitable to store huge amounts of information that would otherwise be erased by environmental noise and decoherence. ${ }^{41}$ It could be proposed that, during the course of evolution, stronger and more sophisticated Maxwell demons (or work extraction mechanisms) have been created and, as a result, more complex matter has been emerged. This continued to the point that living matter was created. Further progress in evolution resulted in the creation of far more advanced Maxwell demons and thus the emergence of conscious matter. Emergence of conscious matter means emergence of consciousness. The QBIT theory suggests that emergence of consciousness is another link in the long chain of matter upgrading events. ${ }^{42}$ If all these conjectures are correct, what is the next stage of the matter upgrading process? A possible next stage is superconscious matter. A superconscious agent might possess not only consciousness and self-consciousness, but also super-consciousness. A creature endowed with super-consciousness could potentially become aware of the 
contents of the minds of other conscious agents. For consciousness, a kind of super-correlation (entanglement) is necessary. For super-consciousness, a kind of superentanglement might be required.

The QBIT theory is a new theory of consciousness in its earliest stage. During the past three decades, several different theories have been proposed and developed to solve the problem of consciousness. This diversity in theories of consciousness should be welcomed and encouraged because it is important for providing insights and driving empirical progress. Two of the most promising and well-developed theories of consciousness are the Integrated Information Theory (IIT) and the Orchestrated Objective Reduction (Orch OR) theory. Although the QBIT theory has borrowed much from these two theories, it introduces some novel ideas and concepts for a scientific study of consciousness.

A similarity between IIT and the QBIT theory is that both theories are constructed on the basic concept of information. The concept of integrated information can in principle be extended to include quantum information because there are interesting parallels between integrated information and principles of quantum mechanics. IIT assumes that quantum entanglement and integrated information are informationally one. $^{43}$

The main similarity between the Orch OR and the QBIT theories is that both are constructed on the basis of quantum mechanics. Both theories propose that consciousness requires 
quantum computation, entanglement, and coherence. The Orch OR theory suggests that, for consciousness to occur in a system, it is necessary that a sufficient amount of material (e.g. microtubule) be kept in a coherent (or pure) state for a long enough time so that objective reduction can be used by the system in a controlled way. ${ }^{44}$ The theory suggests that different states of a tubulin represent information in the brain. It considers tubulin bits (and quantum bits, or qubits) as entangled coherent states. These coherent states of microtubules in one neuron can extend by entanglement to microtubules in adjacent neurons, potentially extending to brain-wide syncytia. ${ }^{44}$

The QBIT theory of consciousness attempts to be dynamic in the sense of being revised and updated repeatedly following accumulation of relevant empirical evidence. Ideas proposed in this first version of the theory might seem to be very far from the mainstream of consciousness studies. However, it should be remembered that, in the history of science, many conjectures initially regarded as being unrealistic ended up being correct explanations.

\section{References}

1. Poznanski RR, Cacha LA, Latif AZA, Salleh SH, Ali J, Yupapin P, Tuszynski JA, Tengku MA. Theorizing how the brain encodes consciousness based on negentropic entanglement. J Integr Neurosci. 2019;18(1):1-10. 
2. Hayden P. Quantum information: putting certainty in the bank. Nature. 2005;436(7051):633-634.

3. Adami C. What is complexity? BioEssays. 2002;24:10851094.

4. Adami C. Information theory in molecular biology. Phys Life Rev. 2004;1:3-22.

5. Adami C. What is information? Phil Trans $\mathrm{R}$ Soc A. 2016;374:20150230.

6. Cerf NJ, Adami C. Negative Entropy and Information in Quantum Mechanics. Phys Rev Lett. 1997;79(26):51945197.

7. Viola L, Knill E, Lloyd S. Dynamical Decoupling of Open Quantum Systems. Phys Rev Lett. 1999;82:24172421.

8. Zhang J, Wu R-B, Li C-W, Tarn T-J. Protecting Coherence and Entanglement by Quantum Feedback Controls. IEEE Transac Autom Cont. 2010;55:619-633.

9. Engel GS, Calhoun TR, Read EL, Ahn TK, Mancal T, Cheng YC, Blankenship RE, Fleming GR. Evidence for wavelike energy transfer through quantum coherence in photosynthetic systems. Nature. 2007; 446(7137):782786.

10. Gauger EM, Rieper E, Morton JJ, Benjamin SC, Vedral V. Sustained quantum coherence and entanglement in the avian compass. Phys Rev Lett. 2011;106(4):040503.

11. Dehaene S, Lau H, Kouider S. What is consciousness, and could machines have it? Science. 2017;358(6362):486-492. 
12. Horodecki R, Horodecki P, Horodecki M, Horodecki K. Quantum entanglement. Rev Mod Phys. 2009;81(2):865942.

13. Cirac JI, Ekert AK, Huelga SF, Macchiavello C. Distributed quantum computation over noisy channels. Phys Rev A. 1999;59:4249.

14. Hameroff SR. The Brain Is Both Neurocomputer and Quantum Computer. Cogn Sci. 2007;31(6):1035-1045.

15. Levine H, Keesling A, Omran A, Bernien H, Schwartz S, Zibrov AS, Endres M, Greiner M, Vuletić V, Lukin MD. High-Fidelity Control and Entanglement of RydbergAtom Qubits. Phys Rev Lett. 2018;121(12):123603.

16. Biamonte J, Wittek P, Pancotti N, Rebentrost P, Wiebe N, Lloyd S. Quantum machine learning. Nature. 2017;549(7671):195-202.

17. August M, Ni X. Using recurrent neural networks to optimize dynamical decoupling for quantum memory. Phys Rev A. 2017;95:012335.

18. Horodecki M, Oppenheim J. Fundamental limitations for quantum and nanoscale thermodynamics. Nat Commun. 2013;4:2059.

19. Lebedev AV, Lesovik GB, Vinokur VM, Blatter G. Extended quantum Maxwell demon acting over macroscopic distances. Phys Rev B. 2018;98:214502.

20. Tu Y. The nonequilibrium mechanism for ultrasensitivity in a biological switch: Sensing by Maxwell's demons. Proc Natl Acad Sci USA. 2008;105(33):11737-11741. 
21. Ito S, Sagawa T. Maxwell's demon in biochemical signal transduction with feedback loop. Nat Commun. 2015;6:7498.

22. Orpwood R. Neurobiological mechanisms underlying qualia. J Integr Neurosci. 2007;6(4):523-540.

23. Beer RD. The Dynamics of Active Categorical Perception in an Evolved Model Agent. Adaptive Behav. 2003;11(4):209-243.

24. Marstaller L, Hintze A, Adami C. The Evolution of Representation in Simple Cognitive Networks. Neural Comput. 2013;25:2079-2107.

25. Kourtzi Z, Kanwisher N. Representation of Perceived Object Shape by the Human Lateral Occipital Complex. Science. 2001;293:1506-1509.

26. Kourtzi Z, Erb M, Grodd W, Bülthoff $\mathrm{HH}$. Representation of the Perceived 3-D Object Shape in the Human Lateral Occipital Complex. Cereb Cortex. 2003;13(9):911-920.

27. Roe AW, Chelazzi L, Connor CE, Conway BR, Fujita I, Gallant JL, Lu H, Vanduffel W. Toward a Unified Theory of Visual Area V4. Neuron. 2012;74(1):12-29.

28. Dehaene S, Changeux J-P. Experimental and theoretical approaches to conscious processing. Neuron. 2011;70(2):200-227.

29. Mai A-T, Grootswagers T, Carlson TA. In search of consciousness: Examining the temporal dynamics of conscious visual perception using MEG time-series data. Neuropsychologia. 2019;129:310-317. 
30. Seevinck MP. Monogamy of correlations versus monogamy of entanglement. Quantum Inf Process. 2010;9(2):273-294.

31. Doherty AC. Entanglement and the shareability of quantum states. J Phys A: Math Theor. 2014;47:424004.

32. Susskind L, Zhao Y. Teleportation through the wormhole. Phys Rev D. 2018;98:046016.

33. Luo S. Wigner-Yanase Skew Information and Uncertainty Relations. Phys Rev Lett. 2003;91:180403.

34. Qi XL. Does gravity come from quantum information? Nat Phys. 2018;14:984-987.

35. Wen X-G. Choreographed entanglement dances: Topological states of quantum matter. Science. 2019;363(6429):eaal3099.

36. Masanes L, Müller MP, Augusiak R, Pérez-García D. Existence of an information unit as a postulate of quantum theory. Proc Natl Acad Sci U S A. 2013;110(41):16373-16377.

37. DiVincenzo DP, Loss D. Quantum information is physical. Superlattices Microstruct. 1998;23(3-4):419432.

38. Banks WP. How Much Work Can a Quale Do? Conscious Cogn. 1996;5(3):368-380.

39. Schossau J, Adami C, Hintze A. Information-Theoretic Neuro-Correlates Boost Evolution of Cognitive Systems. Entropy. 2016;18(1):6. 
40. Michel D. Basic statistical recipes for the emergence of biochemical discernment. Prog Biophys Mol Biol. 2011;106(3):498-516.

41. Ribezzi-Crivellari M, Ritort F. Large work extraction and the Landauer limit in a continuous Maxwell demon. Nat Phys. 2019. DOI: 10.1038/s41567-019-0481-0

42. Beshkar M. A thermodynamic approach to the problem of consciousness. Med Hypotheses. 2018;113:15-16.

43. Tononi G. Consciousness as Integrated Information: a Provisional Manifesto. Biol Bull. 2008;215(3):216-242.

44. Hameroff S, Penrose R. Consciousness in the universe: A review of the 'Orch OR' theory. Phys Life Rev. 2014;11(1):39-78. 